\title{
Visco-elastic systems as a quadratic eigenvalue problem
}

\author{
Maria Antónia Forjaz*, António Mário Almeida ${ }^{\dagger}$, Luís M. Fernandes ${ }^{* *}$, Jorge \\ Pamplona ${ }^{\ddagger}$ and T. de Lacerda-Arôso ${ }^{\dagger}$ \\ ${ }^{*}$ Centre of Mathematics, University of Minho \\ ${ }^{\dagger}$ Centre of Physics, University of Minho \\ ** Instituto Politécnico de Tomar e Instituto de Telecomunicações, Coimbra, Portugal \\ ${ }^{\sharp}$ Institute of Earth Sciences (ICT), University of Minho
}

\begin{abstract}
.
In viscous materials systems, time and stress dependent instabilities often occur. The evolution of visco-elastic systems under external stress has already been modeled by applying matricial dynamic equations comprehending elasticity and viscosity matrices. In this study we report a novel formulation for such kind of systems as a nonlinear quadratic eigenvalue problem evolving from an already defined adjacency matrix. A four mass-spring damped system is presented as case study.

Keywords: quadratic eigenvalue problem, visco-elastic systems, damped mass-spring system
\end{abstract}

PACS: $02.60 . \mathrm{Cb}$

\section{INTRODUCTION}

The harmonic oscillator is the paradigm to all condensed matter. In a structured material system, resonance occurs when the structure is excited by external forces whose frequencies mime the natural frequencies/modes of the system itself. Then, the system vibrations are amplified towards infinity and it becomes unstable. The natural modes of a structure can be seen as the solution of an eigenvalue problem, that is quadratic when damping effects are included in the model.

When considering the evolution of a damped visco-elastic systems under external stress, it may be identified as a nonlinear quadratic eigenvalue problem which models the second order differential equation of the momentum balance of the system. This was confirmed by the modularization of an interconnected 2D damped mass-spring system with eight degrees of freedom, for which the solution of the dynamic equation was successfully applied as herein presented.

After briefly addressing the problem of damped visco-elastic systems under external stress and introducing its physical dynamic equation, it is addressed as a quadratic eigenvalue problem. For this formulation new contributions were developed. A case study of a four mass-spring damped system is analyzed as a QEP, calling upon MatLab.

\section{QUADRATIC EIGENVALUE PROBLEM}

Under an external applied $F$ the dynamics of a system is governed by the momentum balance equation (Newton second law). Considering elasticity and viscosity, it follows:

$$
M \ddot{u}(t)+B \dot{u}(t)+K u(t)=F(t)
$$

where $M$ is the mass matrix (symmetric and positive definite), $K$ is the elasticity matrix (positive definite), $B$ is the viscosity matrix (symmetric), and $u(t)$ stands for the $n$ point masses individual displacements. The (static) resistance to displacement is provided by a spring of elasticity $K$, while the (dynamic) energy loss mechanism is represented by a damper $B$ and $F(t)$ represents the external force.

The general solution to the homogeneous equation has the form:

$$
u(t)=v e^{\lambda t}
$$

where $\lambda$ and $v$ are a scalar and a vector, respectively. 
The solution of the dynamic equation (1) can be expressed as a nonlinear eigenvalue problem in terms of the eigensolution of the corresponding Quadratic Eigenvalue Problem (QEP).

The Quadratic Eigenvalue Problem has extensive applications in areas such as the dynamic analysis of structures with proportional damping models, electrical circuit simulation or linear stability of flows in fluid mechanics. The review paper by Tisseur and Meerbergen [1] describes many applications of the QEP.

Given $M, B, K \in \mathbb{C}^{r \times r}$, the QEP formal definition consists on finding scalars $\lambda$ and nonzero vectors $v$ and $w$, such that:

$$
\left(\lambda^{2} M+\lambda B+K\right) v=0 \quad \text { and } \quad w^{*}\left(\lambda^{2} M+\lambda B+K\right)=0,
$$

where $v$ and $w$ are the left and the right eigenvectors corresponding to the eigenvalue $\lambda$ [1]. In all, QEP has $2 r$ eigenvalues with up to $2 r$ right and $2 r$ left eigenvectors, though no more than $r$ eigenvectors linearly independent.

In the matrix polynomial of degree 2

$$
Q(\lambda)=\lambda^{2} M+\lambda B+K
$$

the coefficients of the matrix are quadratic polynomials in the scalar $\lambda$.

Matrix $Q(\lambda)$ is self-adjoint if $Q(\lambda)=Q(\bar{\lambda})^{*}$ for all $\lambda \in \mathbb{C}$ or, equivalently, if $M, B$, and $K$ are Hermitian matrices. Knowing that the eigenvalues of a self-adjoint matrix $Q(\lambda)$ are real or arise in complex conjugate pairs:

$$
Q(\lambda) v=0 \Leftrightarrow w^{*} Q(\bar{\lambda})=0
$$

where $v$ is a right eigenvector of $\lambda$ and $w$ is a left eigenvector of $\bar{\lambda}$. When the matrices are real, then the sets of left and right eigenvectors coincide.

When the matrices $M, B$, and $K$ are real and symmetric, $M, B>0$, and $K \geq 0$, since $v$ is an eigenvector, the roots of $v^{*} Q(\lambda) v=0$ are

$$
\lambda=\left(\left(v^{*} B v\right) \pm \sqrt{\left(v^{*} B v\right)^{2}-4\left(v^{*} M v\right)\left(v^{*} K v\right)}\right) /\left(2 v^{*} M v\right),
$$

and we say that the system is overdamped when it is satisfied the overdamping condition:

$$
\min _{\|v\|_{2}=1}\left[\left(v^{*} B v\right)^{2}-4\left(v^{*} M v\right)\left(v^{*} K v\right)\right]>0 .
$$

We observe that for $B>0$ and $K>0$, we have $\operatorname{Re}(\lambda)<0$, all the eigenvalues are real and nonpositive, lying in the left half-plane and the system is stable [1].

\section{A 2-D MODEL OF INTERCONNECTED MASSES}

As an example of a QEP, we consider the interconnected 2D damped mass-spring system illustrated in Figure 1. Each mass has 2 degrees of freedom and is connected to all the others by a spring and a damper with constants $\kappa$ (elasticity) and $b$ (friction or viscous damping), respectively.

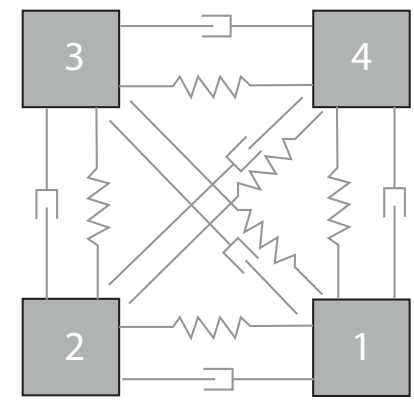

FIGURE 1. An 8-degrees of freedom damped mass-spring system. 
The second order differential equation (1) governs the behavior of the system where the mass matrix $M$ is diagonal a the elasticity and damping matrices, $K$ and $B$ are written according to (2) [2] .

Should we consider the above system as so, with a square geometry (A) (Figure 1) and when slightly distorted into a polygon derived geometry (B). In the latter case, only one mass point was displaced by a small amount along a diagonal.

Assuming all masses $m$ are equal and elastic $\kappa$ and damping $b$ constants are the same for each pair of interconnected masses, we can seek for solutions at the overdamping regime.

The associated mass matrix $M$ is a $2 n \times 2 n$ diagonal, and the elasticity $K$ and damping $B$ matrices are $2 n \times 2 n$ symmetric and defined after [2] as,

$$
K=\kappa\left(\begin{array}{cc|cc}
P & O_{\alpha} & O_{\gamma} & O_{\delta} \\
O_{\alpha} & Q & O_{\beta} & O_{\theta} \\
\hline O_{\gamma} & O_{\beta} & R & O_{\varphi} \\
O_{\delta} & O_{\theta} & O_{\varphi} & S
\end{array}\right), \quad B=b\left(\begin{array}{cc|cc}
P & O_{\alpha} & O_{\gamma} & O_{\delta} \\
O_{\alpha} & Q & O_{\beta} & O_{\theta} \\
\hline O_{\gamma} & O_{\beta} & R & O_{\varphi} \\
O_{\delta} & O_{\theta} & O_{\varphi} & S
\end{array}\right)
$$

where the block sub-matrices $O_{i}(i=\alpha, \beta, \gamma, \theta, \varphi$ and $\delta), \mathrm{P}, \mathrm{Q}, \mathrm{R}$ and $\mathrm{S}$ traduce the geometrical relations between each node and its neighbors. Angles $\alpha, \beta, \gamma, \theta, \varphi$ and $\delta$ result from the direction defined by every couple of nodes relative to the $\mathrm{X}$-axis (see details in [2]).

Taking, for example, the set of parameters $m=1, \kappa=1$ and $b=10$ for both geometries A and B, the above $K$ and $B$ matrices were built and the eigenvalues of equation $Q(\lambda) v=0$ obtained in MatLab, by calling the function $\operatorname{polyeig}(K, B, M)[3]$.

The results are plotted in Figure 2. All the eigenvalues are real and non-positive, as expected for an overdamped regime [1]. The eigenvalues are degenerate for the square geometry (A) though degeneracy is partially broken for geometry B due to its lower symmetry.

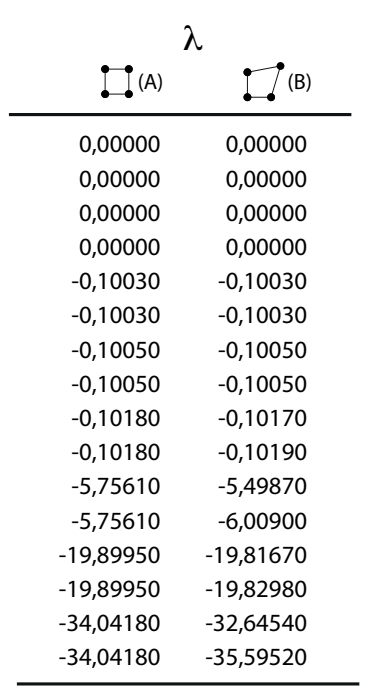

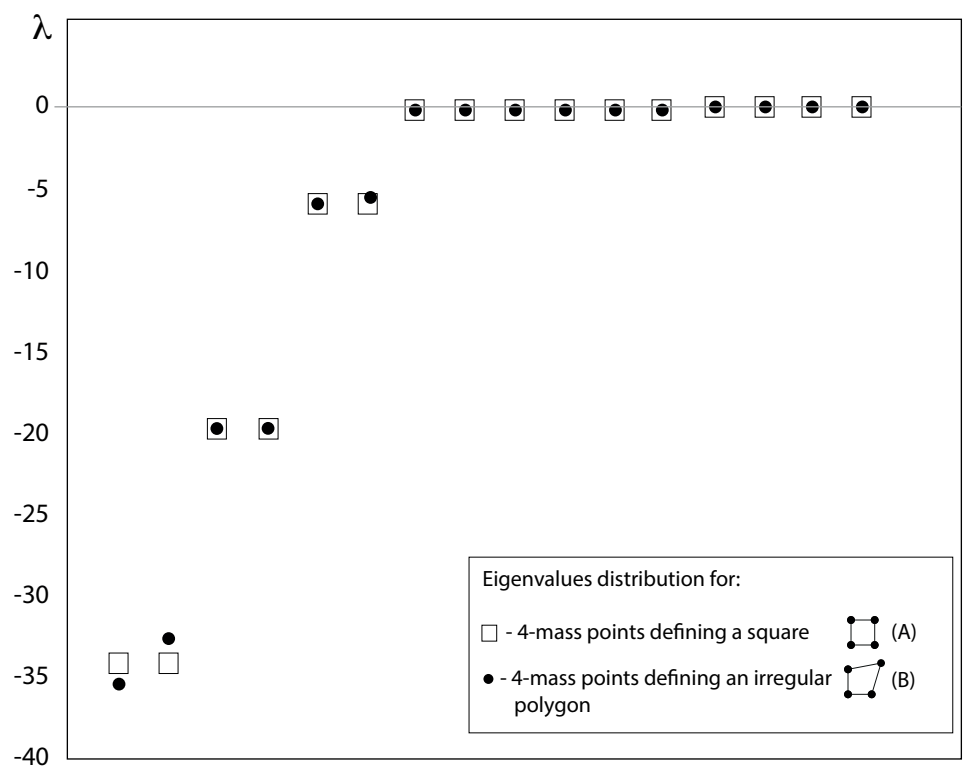

FIGURE 2. Eigenvalue distribution of the QEP for overdamped mass-spring systems $A(\square)$ and B (•).

\section{FINAL REMARKS}

The introduced QEP case can be generalized for an arbitrary large number of disordered point masses, belonging to a single material (characterized by a single set of $m, \kappa$ and $b$ ), or to heterogeneous - blended or layered - materials with different sets of parameters. 
In a previous work [4], we have developed an algorithm, to a domain of material points, that establishes the set of physical bonds between any two neighbours (Figure 3) and their geometrical relations (angles $\alpha, \beta, \gamma, \theta, \varphi$ and $\delta$ ), so to define an adjacency matrix. This is the scaffold to build up matrices $K$ and $B$, used in the herein QEP example.

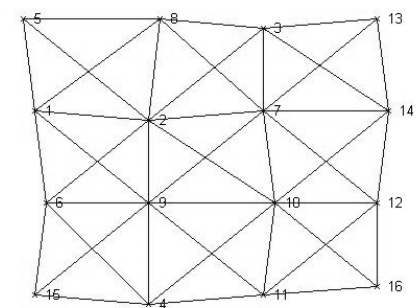

FIGURE 3. Complete set of one-to-one physical bonds in a domain of material points.

As an undamped dynamic system has already been addressed as an Eigenvalue Complementarity Problem (EiCP) [5], we intend to treat a broader case by formulating a Quadratic Eigenvalues Complementarity Problem (QEiCP) [6] to a visco-elastic system.

\section{ACKNOWLEDGMENTS}

The author Maria Antónia Forjaz acknowledges the funding supported by Portuguese Funds through FCT - 'Fundação para a Ciência e a Tecnologia', UID/MAT/00013/2013.

The author Jorge Pamplona is co-funded by the European Union through the European Regional Development Fund, based on COMPETE 2020 (Programa Operacional da Competitividade e Internacionalização), project ICT (UID/GEO/04683/2013) with reference POCI-01-0145-FEDER-007690 and national funds provided by Fundação para a Ciência e Tecnologia.

The authors António Mário Almeida and T. de Lacerda-Arôso acknowledge the support of the Portuguese Foundation for Science and Technology (FCT) in the framework of the Strategic Funding UID/FIS/04650/2013.

\section{REFERENCES}

1. F. Tisseur and K. Meerbergen. The quadratic eigenvalue problem. SIAM Review, Vol. 43, No. 2, pp. 235-286, 2001.

2. M. A. Forjaz, A.M. Almeida, T. de Lacerda-Arôso and J. Pamplona, Complementary Eigenvalue Problem in systems with frictional contact: the Stiffness Matrix for the contact nodes between different materials, in Proceeding of the 14th International Conference on Computational Science and Its Applications (ICCSA 2014), edited by A. M. Rocha. J. G. Rocha and M. I. Falcão (IEEE Computer Society, Guimarães, Portugal, 2014), pp. 268-271.

3. Computer code MATLAB, v. 8.2.0.701 (R2013b). The MathWorks Inc., Natick, Massachusetts (2013).

4. M. A. Forjaz, A.M. Almeida, T. de Lacerda-Arôso and J. Pamplona, Special matrices for visco-elastic systems, In AIP Conference Proceedings of the International Conference on Numerical Analysis and Applied Mathematics 2015 (ICNAAM2015). Edited by Theodore E. Simos and Charalambos Tsitouras.

5. A. Pinto da Costa, I. Figueiredo, J. J. Júdice and J. Martins, A complementarity eigenproblem in the stability analysis of finite dimensional elastic systems with frictional contact, in Complementarity: Applications, Algorithms and Extensions, edited by M.C. Ferris, O.L. Mangasarian and J.S. Pang (Kluwer Academic Publishers, Dordrecht, 2001), pp. 67-83.

6. Fernandes, L.M., Júdice, J., Sherali, H., Fukushima, Alfredo Iusen, On the symmetric quadractic eigenvalues complementarity problems, Optimization Methods and Software 29 (2014) 751 - 770. 